and the prognosis of anti-PL7 ASS under treatment were heterogeneous.

\section{B. Hervier*, Y. Uzunhan", E. Hachulla", O. Benveniste ${ }^{+}$, H. Nunes ${ }^{\#}$, P. Delaval ${ }^{\S}$, L. Musset ${ }^{f}$, S. Dubucquoi**, B. Wallaert ${ }^{\# \#}$ and M. Hamidou*}

*Internal Medicine Dept, CHU Nantes Hotel Dieu, Nantes, "Pneumology Dept, CHU Avicenne, ${ }^{+}$Internal Medicine Dept, CHU Pitie-Salpetriere, French Reference Centre for Myositis, ${ }^{f}$ Laboratory of immunology, CHU Pitie-Salpetriere, Paris, "Internal Medicine Dept, CHRU Lille, **Laboratory of immunology, CHRU Lille, "\#neumology Dept, CHRU Lille, Lille, and ${ }^{\S}$ Pneumology Dept, CHU Rennes, Rennes, France.

Correspondence: B. Hervier, Internal Medicine Dept, CHU Nantes Hotel Dieu, Place Alexis Ricordeau, 44093 Nantes Cedex, France. E-mail: bhervier@yahoo.fr

Statement of Interest: None declared.

\section{REFERENCES}

1 Marguerie C, Bunn CC, Beynon HL, et al. Polymyositis, pulmonary fibrosis and autoantibodies to aminoacyl-tRNA synthetase enzymes. Q J Med 1990; 77: 1019-1038.

2 Fischer A, Swigris JJ, du Bois RM, et al. Anti-synthetase syndrome in ANA and anti-Jo-1 negative patients presenting with idiopathic interstitial pneumonia. Respir Med 2009; 103: 1719-1724.
3 Sato S, Hirakata M, Kuwana M, et al. Clinical characteristics of Japanese patients with anti-PL-7 (anti-threonyl-tRNA synthetase) autoantibodies. Clin Exp Rheumatol 2005; 23: 609-615.

4 Yamasaki Y, Yamada H, Nozaki T, et al. Unusually high frequency of autoantibodies to PL-7 associated with milder muscle disease in Japanese patients with polymyositis/dermatomyositis. Arthritis Rheum 2006; 54: 2004-2009.

5 Mimori T, Imura Y, Nakashima R, et al. Autoantibodies in idiopathic inflammatory myopathy: an update on clinical and pathophysiological significance. Curr Opin Rheumatol 2007; 19: 523-529.

6 Vitali C, Bombardieri S, Jonsson R, et al. Classification criteria for Sjögren's syndrome: a revised version of the European criteria proposed by the American-European Consensus Group. Ann Rheum Dis 2002; 61: 554-558.

7 Devouassoux G, Cottin V, Lioté $\mathrm{H}$, et al. Characterisation of severe obliterative bronchiolitis in rheumatoid arthritis. Eur Respir J 2009; 33: 1053-1061.

8 Tillie-Leblond I, Wislez M, Valeyre D, et al. Interstitial lung disease and anti-Jo-1 antibodies: difference between acute and gradual onset. Thorax 2008; 63: 53-59.

9 Hervier B, Wallaert B, Hachulla E, et al. Clinical manifestations of anti-synthetase syndrome positive for anti-alanyl-tRNA synthetase (anti-PL12) antibodies: a retrospective study of 17 cases. Rheumatology. (Oxford) 2010; 49: 972-976.

10 LeRoy EC, Medsger TA Jr. Criteria for the classification of early systemic sclerosis. J Rheumatol 2001; 28: 1573-1576.

11 Tzelepis GE, Toya SP, Moutsopoulos HM. Occult connective tissue diseases mimicking idiopathic interstitial pneumonias. Eur Respir J 2008; 31: 11-20.

\title{
Obstructive sleep apnoea and metabolic syndrome in Mediterranean countries
}

\section{To the Editors:}

Obstructive sleep apnoea (OSA) is often associated with metabolic disturbances, including altered glucose metabolism and dyslipidaemia, which probably contribute to the increased cardiovascular risk in these patients [1]. The concept of the metabolic syndrome (MetS) as a cluster of cardiometabolic risk factors has gained popularity in recent years, and a much higher prevalence of the MetS has been found in OSA patients compared with the general population in several studies [1]. While the MetS largely reflects the effects of visceral obesity, environmental factors, i.e. the type of diet, could also play some role. The Mediterranean diet, rich in olive oil and fish, is protective against the MetS [2-4], but no study has examined the association of MetS and OSA in Mediterranean countries. We hypothesised that prevalence of the MetS might be lower in OSA patients living in the Mediterranean area compared with the prevalence values found in non-Mediterranean countries. Therefore, we retrospectively assessed the prevalence of the MetS according to the modified National Health and Nutrition Examination Survey Adult Treatment Panel (ATP) III criteria [5] in consecutive patients referred to sleep laboratories in Italy $(n=107)$, Spain $(n=138)$ and Greece $(n=218)$.
Patients diagnosed with OSA in the period July 2007-September 2008 in Palermo, Italy (Respiratory Section, DIBIMIS, University of Palermo, and CNR Institute of Biomedicine and Molecular Immunology), Palma de Mallorca, Spain (Hospital Son Dureta) and Alexandroupolis, Greece (Sleep Unit, Medical School, Democritus University of Thrace), were evaluated in this study. All underwent clinical examination for clinical suspicion of OSA, and full polysomnography or nocturnal cardiorespiratory monitoring (eight channel) according to the American Academy of Sleep Medicine guidelines [6]. OSA was diagnosed when the apnoea/hypopnoea index (AHI) was $>5$ events $\cdot h^{-1}$; mean lowest arterial oxygen saturation $\left(\mathrm{Sa}_{1} \mathrm{O}_{2}\right)$ was recorded. Daytime sleepiness was subjectively assessed by the Epworth Sleepiness Score questionnaire. Body mass index (BMI) was defined as $\mathrm{kg} \cdot \mathrm{m}^{-2}$. Neck, waist and hip circumferences $(\mathrm{cm})$ were measured. The MetS was diagnosed based on the presence of three or more of the following factors: waist circumference $\geqslant 80 \mathrm{~cm}$ in females and $\geqslant 94 \mathrm{~cm}$ in males; serum triglycerides $\geqslant 150$ $\mathrm{mg} \cdot \mathrm{dL}^{-1}$ or lipid-lowering treatment; high-density lipoprotein $\mathrm{HDL}$ cholesterol $<40 \mathrm{mg} \cdot \mathrm{dL}^{-1}$ in males and $<50 \mathrm{mg} \cdot \mathrm{dL}^{-1}$ in females or statin treatment; systemic hypertension (systolic blood pressure $>135 \mathrm{mmHg}$ and/or diastolic blood pressure $>85 \mathrm{mmHg}$ ) or anti-hypertensive treatment; and fasting blood 
glucose $>100 \mathrm{mg} \cdot \mathrm{dL}^{-1}$ or anti-diabetic treatment [5]. Plasma glucose and lipids were analysed by standard laboratory methods in venous blood withdrawn in the morning in a fasting state. Treatment for hypertension (i.e. diuretics, $\beta$-blockers and angiotensin-converting enzyme (ACE) inhibitors, etc.), diabetes (i.e. oral antidiabetic drugs and insulin), or dyslipidaemia (i.e. statins and clofibrate, etc.) was recorded in all patients.

Data are reported as mean \pm SD for continuous variables, and percentage of positive subjects for categorical variables. Continuous variables in the three patients' samples were compared by one-way ANOVA with Bonferroni correction for multiple comparisons. The Chi-squared test was used to assess differences in the frequency of categorical variables. Variables significantly associated with MetS were tested by multiple logistic regression. Statistical analysis was performed by using the SPSS version 17 software (SPSS Inc., Chicago, IL, USA) and significance was at $\mathrm{p}<0.05$ for all tests.

A total of 463 patients were diagnosed with OSA in the study period (107 in Palermo, 218 in Alexandroupolis and 138 in Palma de Mallorca), but 127 had to be excluded due to missing information (20, 98 and nine, from the respective samples), leaving 336 patients for analysis. Excluded patients did not show any significant difference compared to included ones for sex distribution, age, BMI or OSA severity (data not shown).

Table 1 summarises the anthropometric and clinical features of the samples. The percentage of female patients was similar in Italy and Spain (between 30\% and 35\%), and lowest in the Greek sample (19\%). Age and AHI were similar in the three groups. Greek patients showed a significantly lower $\mathrm{Sa}_{\mathrm{a}} \mathrm{O}_{2}$ during sleep. Mean BMI and waist circumference were highest in the Greek patients, who also showed significantly higher treatment rates for hypertension (ACE inhibitors 34.2\%, $\beta$-blockers $11.7 \%$ and diuretics $19.2 \%$ ), diabetes (oral hypoglycemic drugs $15.8 \%$ ), or dyslipidaemia $(29.2 \%)$ compared to Italian patients (ACE inhibitors $17.2 \%$, $\beta$-blockers $8.0 \%$, diuretics $6.9 \%$, oral hypoglycemic drugs $3.4 \%$ and dyslipidaemia treatment $9.2 \%$ ), or Spanish patients (ACE inhibitors 14.2\%, $\beta$-blockers $7.4 \%$, diuretics $16.8 \%$, oral hypoglycemic drugs $7.4 \%$ and dyslipidaemia treatment $11.9 \%$ ). Waist-to-hip ratio was lowest in Italian patients, intermediate in Greek patients and highest in Spanish patients.

According to the National Cholesterol Education Program (NCEP)-ATP III definition, MetS was present in $67.3 \%$ of the patients of the entire cohort (fig. 1 and table 1), with the lowest prevalence in the Italian sample $(p<0.0001$ by Chi-squared test). Significant differences in the prevalence of single MetS components were found among the samples for waist circumference, triglycerides and fasting blood glucose, while prevalence of hypertension or low HDL-cholesterol was similar in the three samples. Logistic regression analysis in the entire sample identified age, BMI, male sex, lowest $\mathrm{Sa}_{2} \mathrm{O}_{2}$ and presence of daytime sleepiness as significantly associated with MetS.

The results of this retrospective study do not support the hypothesis that patients with OSA from Mediterranean countries may be protected against MetS. Indeed, the prevalence rates observed were similar to those reported by other clinical studies in Western countries ranging between 30\% and $87 \%$ [1]. Prevalence of MetS in Mediterranean OSA patients was at least twice as high as in the general population of the respective countries, which is rising and currently estimated to be between $22 \%$ and $25 \%$ [7-9]. The Greek sample showed the highest prevalence of markers of abdominal obesity, accounting

TABLE 1 Characteristics of the patients

\begin{tabular}{|c|c|c|c|c|c|}
\hline & All patients & $\begin{array}{l}\text { Palermo, } \\
\text { Italy }\end{array}$ & $\begin{array}{c}\text { Alexandroupolis, } \\
\text { Greece }\end{array}$ & $\begin{array}{c}\text { Palma de Mallorca, } \\
\text { Spain }\end{array}$ & $\mathrm{p}$-value \\
\hline Males \% & 72.3 & 65.5 & 80.8 & 69.0 & $<0.05$ \\
\hline Age yrs & $55.3 \pm 12.3$ & $54.3 \pm 11.1$ & $57.3 \pm 12.8$ & $54.2 \pm 12.4$ & NS \\
\hline$B M I \mathbf{k g} \cdot \mathrm{m}^{-2}$ & $33.0 \pm 7.2$ & $33.0 \pm 7.5$ & $34.4 \pm 6.4$ & $31.7 \pm 7.6$ & $<0.01^{\#}$ \\
\hline Lowest nocturnal $\mathrm{Sa}, \mathrm{O}_{2} \%$ & $78.4 \pm 11.7$ & $81.9 \pm 11.5$ & $73.5 \pm 12.6$ & $80.3 \pm 9.2$ & $<0.001^{\circ}$ \\
\hline Epworth Sleepiness Scale score & $9.6 \pm 5.4$ & $9.9 \pm 5.4$ & $9.7 \pm 5.7$ & $9.4 \pm 5.1$ & NS \\
\hline Waist-to-hip ratio & $1.00 \pm 0.09$ & $0.95 \pm 0.07$ & $1.00 \pm 0.07$ & $1.03 \pm 0.10$ & $<0.001^{+}$ \\
\hline Waist circumference $\mathrm{cm}$ & $112.0 \pm 14.9$ & $108.3 \pm 16.2$ & $117.6 \pm 14.7$ & $108.7 \pm 12.7$ & $<0.0001^{\natural}$ \\
\hline SBP mmHg & $130.9 \pm 17.8$ & $131.5 \pm 18.6$ & $129.7 \pm 19.1$ & $131.5 \pm 16.4$ & NS \\
\hline DBP mmHg & $81.9 \pm 10.6$ & $84.7 \pm 12.3$ & $81.4 \pm 8.2$ & $81.1 \pm 11.2$ & NS \\
\hline Prevalence of MetS \% & 67.3 & 50.6 & 76.7 & 69.8 & $<0.0001$ \\
\hline
\end{tabular}

Data are presented as mean \pm SD, unless otherwise stated. BMI: body mass index; AHI: apnoea/hypopnoea index; $\mathrm{Sa}, \mathrm{O}_{2}$ : arterial oxygen saturation; SBP: systolic blood pressure; DBP: diastolic blood pressure; HDL: high-density lipoprotein; MetS: metabolic syndrome; NS: nonsignificant. Results of multiple comparison testing: \#: Greece $>$ Spain; ${ }^{*}$ : Greece $>$ Italy and Spain; ${ }^{+}$: Spain > Greece > Italy; ${ }^{\text {s. }}$ Spain > Italy. 


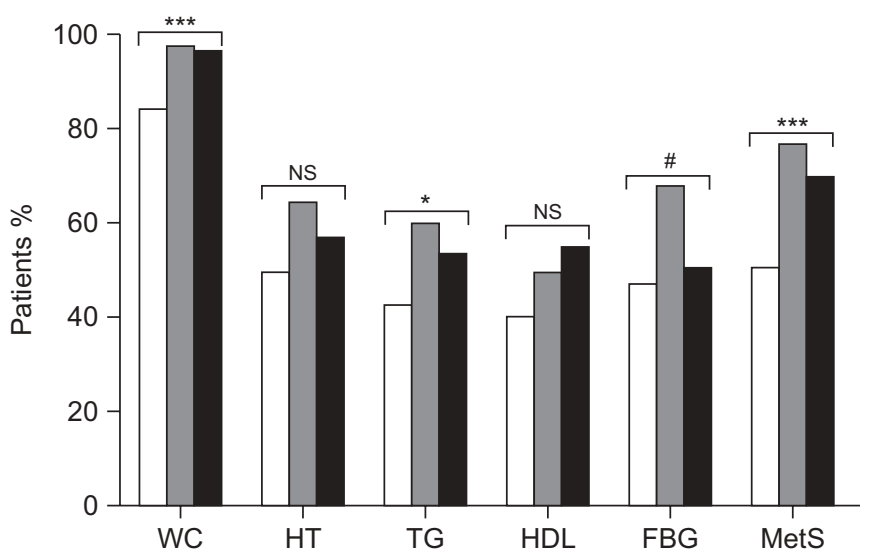

FIGURE 1. Metabolic syndrome (MetS) and its components. Significant differences in the prevalence of MetS were found among the samples for waist circumference (WC), triglycerides (TG) and fasting blood glucose (FBG). Hypertension (HT) and low high-density lipoprotein (HDL)-cholesterol were similar in the three samples. $\square$ : Italy; $\square$ : Greece; $\mathbf{\square}$ : Spain. Ns: nonsignificant. *: $p<0.05$; $* * *: p<0.001 ; *: p<0.005$

for the highest prevalence of MetS. The lowest prevalence of MetS was found in Southern Italy, together with the highest percentage of female patients and a relatively low waist-to-hip ratio. The Italian sample was quite similar to the Spanish one for percentage of females and waist circumference, but prevalence of MetS was almost 20\% higher in Spain than in Italy. This finding leaves the possibility that some differences in diet may play a role, but we could not check for dietary composition in this retrospective study. Epidemiological data from Spain recently showed that prevalence of MetS in the general population by NCEP-ATP III criteria increased from $18 \%$ to $25 \%$ in a decade, especially in young subjects, in conjunction with a major shift from the Mediterranean diet towards the Western diet [10]. Our study suggests that such a change might have similarly occurred in patients with OSA, although significance of severity of hypoxaemia and sleepiness by logistic regression analysis confirms a possible role of intermittent hypoxia in the pathogenesis of metabolic disorders. In conclusion, prevalence of the MetS in patients with OSA was similar in Mediterranean and non-Mediterranean countries, suggesting that exposure to Mediterranean diet was either absent in these patients or insufficient to exert any protective effect. Studies on the dietary habits of OSA patients are warranted, especially regarding diet composition, as they may be useful in weight control and cardiovascular prevention programmes.

\section{A. Zito*, P. Steiropoulos", A. Barceló ${ }^{\ddagger}$, O. Marrone ${ }^{+}$, C. Esquinas ${ }^{\S}$, M. Buttacavoli*, F. Barbé ${ }^{f * * *}$ and M.R. Bonsignore ${ }^{*,+}$}

*Biomedical Dept of Internal and Specialistic Medicine (DIBIMIS), Respiratory Section, University of Palermo, and ${ }^{+}$Institute of Biomedicine and Molecular Immunology (IBIM), National Research Council (CNR), Palermo, Italy. *Sleep Unit, Medical School, Democritus University of Thrace,
Alexandroupolis, Greece. 'Clinical Analysis Service, Hospital Universitari Son Dureta, Palma de Mallorca, ${ }^{\S}$ Respiratory Disease Unit, Hospital Santa Maria, ${ }^{f}$ Respiratory Disease Research Unit, IRB Lleida, Lleida, and ${ }^{* *}$ Centro de Investigación Biomédica en Red de Enfermedades Respiratorias (CIBERES), Spain.

Correspondence: M.R. Bonsignore, Biomedical Dept of Internal and Specialistic Medicine (DIBIMIS), Respiratory Section, University of Palermo, Via Trabucco 180, 90146 Palermo, Italy. E-mail: marisa@ibim.cnr.it

Statement of Interest: None declared.

Acknowledgements: This work was conceived and accomplished by researchers participating in the European Union-funded COST Action B26 on OSA and cardiovascular risk.

\section{REFERENCES}

1 Lévy P, Bonsignore MR, Eckel J. Sleep, sleep-disordered breathing and metabolic consequences. Eur Respir J 2009; 34: 243-260.

2 Tortosa A, Bes-Rastrollo M, Sanchez-Villegas A, et al. Mediterranean diet inversely associated with the incidence of metabolic syndrome: the SUN prospective cohort. Diabetes Care 2007; 30: 2957-2959.

3 Rumawas ME, Meigs JB, Dwyer JT, et al. Mediterranean-style dietary pattern, reduced risk of metabolic syndrome traits, and incidence in the Framingham Offspring Cohort. Am J Clin Nutr 2009; 90: 1608-1614.

4 Paletas K, Athanasiadou E, Sarigianni M, et al. The protective role of the mediterranean diet on the prevalence of metabolic syndrome in a population of Greek obese subjects. J Am Coll Nutr 2010; 29: 41-45.

5 Alberti KG, Eckel RH, Grundy SM, et al. Harmonizing the metabolic syndrome: a joint interim statement of the International Diabetes Federation Task Force on Epidemiology and Prevention; National Heart, Lung, and Blood Institute; American Heart Association; World Heart Federation; International Atherosclerosis Society; and International Association for the Study of Obesity. Circulation 2009; 120: $1640-1645$.

6 Iber C, Ancoli-Israel S, Chesson A, Quan SF. The AASM Manual for the Scoring of Sleep and Associated Events: Rules, Terminology, and Technical Specifications. 1st Edn. Westchester, American Academy of Sleep Medicine, 2007.

7 Buckland G, Salas-Salvadó, Roure E, et al. Sociodemographic risk factors associated with metabolic syndrome in a Mediterranean population. Public Health Nutr 2008; 11: 1372-1378.

8 Noto D, Barbagallo CM, Cefalù AB, et al. The metabolic syndrome predicts cardiovascular events in subjects with normal fasting glucose: results of a 15 years follow-up in a Mediterranean population. Atherosclerosis 2008; 197: 147-153.

9 Athyros VG, Bouloukos VI, Pehlivanidis AN, et al. The prevalence of the metabolic syndrome in Greece: the MetS-Greece multicentre study. Diabetes Obes Metabol 2005; 7: 397-405.

10 Buckland GG, Salas-Salvadó J, Serra-Majem L, et al. Increase in metabolic syndrome as defined by ATPIII from 1992-1993 to 20022003 in a Mediterranean population. Nutrition Rev 2009; 67: Suppl. 1, S117-S125. 